\title{
GHRONIC PROGRESSIVE OPHTHALMOPLEGIA OF MYOPATHIC ORIGIN
}

\author{
BY \\ P. H. SANDIFER \\ (RECEIVED 23RD JULy, 1946)
}

THE term "chronic progressive ophthalmoplegia" signifies a syndrome characterized by a slowly progressive weakness of the external ocular muscles. In the past the term has been applied loosely, for cases have been called chronic progressive ophthalmoplegia which differ from each other in important respects. Thus, in some instances the paralysis has been confined to the levators of the upper lids (Fuchs, 1890, and Collins, 1909); in others complete external ophthalmoplegia has been present, notably in the cases of Beaumont (1900), McMullen (1912), Altland (1909), McMullen and Hine (1921). Usually the intrinsic eye muscles escape, but this is not always the case. Sometimes there has been associated weakness of the orbicularis oculi (Beaumont, 1900) and of other muscles of the face and of the limbs (McMullen and Hine 1921). In some there has been no family history, whilst in others the disease has been hereditary (Ayres, 1896 ; Cooper, 1910 ; Bradburne, 1912 ; Crouzon, 1929). The scanty pathological material has shown that the oculomotor nuclei may be the site of degenerative changes in some instances but not in others. In 1900 Wildbrand and Saenger grouped together certain cases of external ophthalmoplegia because they believed them to have characteristics which separated them from a congenital type on the one hand and a type symptomatic of neurological disease extending beyond the oculo-motor territory on the other. This group, which they separated, they believed formed a clinical entity which had the following characteristics : the onset was usually in infancy or early childhood; the progress was very slow, extending over periods as long as $\mathbf{3 0}$ or $\mathbf{4 0}$ years ; ptosis was usually the first sign, to be followed by increasing restrictions of the external ocular movements, typically without diplopia; the paralysis might halt for long periods, but as a rule complete fixation of the eyeballs was the final state, though the ptosis was seldom complete. The pathology underlying the symptoms of this group still remains a problem. Nuclear degenerative changes have been thought to be the cause of the paralysis, but in the absence of conclusive proof, notably by Cooper (1910) and Crigler (1914). The term "chronic progressive nuclear ophthalmoplegia" has been applied to those cases showing a slowly increasing oculo-motor palsy which is presumed to be due to degeneration of the cells of the 3rd, 4th, and 6th cranial nerve nuclei. The few published accounts of this condition have been reviewed by Wildbrand and Saenger (1900) : in some of the cases studied degenerative changes have been found in the oculo-motor nuclei; but even so it has been impossible to determine if the degeneration has been primary, or secondary, to some other disease process.

The syndrome of gradually increasing oculo-motor paralysis may certainly be produced in several ways. It may be the first sign of disease involving muscles or neuro-muscular junctions, as may be the case in myotonia atrophica, progressive spinal muscular atrophy (Werdnig-Hoffman's disease), and myasthenia gravis. It may be an early manifestation of such conditions as Parkinsonism, tabes dorsalis, or syringobulbia. To avoid confusion, care should be taken in signifying the groups which Wildbrand and Saenger have distinguished. Where the syndrome is symptomatic and is witnessed in a setting of some well-recognized disease the symptoms of which extend beyond the oculo-motor muscles, the term " chronic progressive ophthalmoplegia" should not be used unless qualified by the name of the disease of which it forms a part. Classification of cases of chronic progressive ophthalmoplegia is not a mere academic exercise but a practical one. The prognosis in that group of chronic progressive ophthalmoplegia which Wildbrand and Saenger separated is favourable in so far as the symptoms remain confined to the oculo-motor muscles. It is of value to the patient and his relatives to learn the probable course of the disease. Unfortunately it is seldom possible to be sure of the category into which a given case should be placed. At present, classification depends largely on the presence or absence of signs outside the territory supplied by the oculo-motor nerves, and upon the patient's personal and family history. However, biopsy may sometimes provide information which is of value in diagnosis. This has been so in the case to be described, which has been interpreted as being an example of chronic progressive ophthalmoplegia of myopathic origin, the evidence being based on histological as well as clinical observation.

\section{Case Record}

A man, aged 29, was admitted to Mount Vernon Hospital as an air-raid casualty on June 30,1944 . He had 
superficial lacerations over the right eyebrow but no other injury... The reason for his admission was that the pulse-rate was 30 per minute. He made no complaints save of his trivial wound, but he admitted that his peculiar appearance produced by his drooping eyelids had been present for many years, as had trouble in moving his eyes. Neither this nor any other symptom prevented him from working as a labourer. He admitted to no weakness of his limb or trunk muscles, neither did he have dyspncea.

His mother said that at the age of 14 his eyelids began to droop. The patient remembered this drooping certainly before the age of 20 . It had always been symmetrical and had gradually progressed. He thought that the ptosis and inability to move the eyes had been stationary for the last 5 years. He denied ever having seen double, although for at least 10 years the eyeballs had become increasingly immobile. His parents were unrelated and there was no story of ptosis nor of weakness in the muscles of the eye or elsewhere, nor of cataract in parents, siblings, or other members of the family.

He was a thin man with poor muscular development but without any muscular wasting or paresis save in certain muscles specified below. Thus he showed gross bilateral ptosis with compensatory contraction of the frontalis muscles. There was, in addition, almost complete fixation of the eyeballs, so that he had to turn the whole head in following a moving object and in reading. There was no internal ophthalmoplegia. The only visual disturbance was defective stereoscopic vision. On testing the facial muscles it was noticed that the orbicularis oculi was weak, so that on tightly screwing up the eyes they could be opened with moderate ease. No other muscles were noted to be weak. In the arms the tendon jerks were absent, but they were normally brisk and symmetrical in the legs. No sensory disturbance of any kind was discovered. There was no improvement of the eye movement after an intramuscular injection of prostigmin. There was no myotonia as judged by percussion of the muscles of the thenar eminence and of the tongue, or by testing the speed of relaxation after powerful and sustained muscular contraction. The distribution of hair was everywhere normal, and the testes were of normal size. The blood Wassermann reaction was negative.

During his stay in hospital the pulse-rate varied between 20 and 66 per minute, on most occasions being about 32. The apex rate was usually about 50 , extra beats being audible as premature systoles. Dr. J. E. G. Pearson found a soft systolic murmur at the apex, with reduplication of the pulmonary second sound. There was no evidence of cardiac decompensation. The electrocardiogram showed very slow irregular rhythm without any normal waves. All QRS waves were wide, suggesting branch bundle block. In lead 2 the beats were in couples, though different. In lead 3 there was a rapid succession of 3 abnormal ventricular complexes and one very queer ectopic ventricular beat after a long pause. No auricular waves were seen. The arm-tongue circulation time, tested by intravenous injection of decholin, was $20 \mathrm{sec}$.

Mr. Maurice Whiting was asked to see the patient with a view to correcting the ptosis and at the same time removing a portion of eye muscle for histological study. Portions of the external recti were removed and the degree of ptosis reduced by surgical means. Dr. J. G. Greenfield reported on the histology of the tissues removed :

"The muscle was cut in paraffin. Owing to the small size of the piece of muscle received, and its atrophic and fibrotic nature, it was not possible to orientate the tissue for section ; but in sections it was found that one area of muscle fibre was cut in longitudinal, and another in transverse section. Histological examination showed some fairly small groups of fibres spaced at a rather greater interval than normal from one another by thin areolar tissue but without any definite interstitial fibrosis. The groups of fibres were, however, separated from one another by thick septa of loosely set collagen fibres. The nerve bundles appeared to be as numerous as normal and showed no evidence of disease or atrophy.

"The healthy muscle fibres were all of about the same size varying from 15 to $30 \mu$, but chiefly were between 20 and $25 \mu$. Very sparse atrophied fibres of $10 \mu$ or less were seen, but there were a number of larger fibres in a state of granular disintegration. These measured up to $37 \cdot 5 \mu$. In these the cytoplasm was granular, with no evidence of striation, and the nuclei were increased in number and rather irregular in shape, and many lay in the middle of the fibre. The outline of these fibres was very irregular (Figs. 1 and 2). Other degenerated fibres were present in which the cytoplasm was irregularly shrunken, was stained more deeply than normal, and contained many large, darkly stained nuclei, which sometimes lay closely opposed in clumps or in pairs (Fig. 3). These nuclei were not evenly set along the length of the fibres, but the groups were separated by a considerable interval of glassy or granular cytoplasm in which no cross striations could be discerned. In cross-sections these nuclei were seen to lie within the muscle fibre and not immediately under the sarcolemma. In longitudinal section the outline of the fibre was irregular, some parts appearing clearer, more granular, and wider in diameter, others shrunken and staining diffusely and rather darkly, i.e. they were more hæmatoxophilic than normal fibres. These granular and hyaline degenerative changes appeared, therefore, to be stages in the same process, both could sometimes be seen in different parts of ths same fibre. Various gradations between a normat appearance and these degenerative changes were seen. Many fibres, for example, had a more solid central corein which transverse striations were still visible although not so marked as in normal fibres-and a looser, rather granular peripheral zone. Some nuclear proliferation was usually seen in such fibres.

"The appearances indicate a myopathic degeneration rather than a neuropathic atrophy. Not only were there extremely few of the very thin fibres seen in neuropathetic atrophy, but the nerves appeared to be quite normal and abundant. On the other hand the degeneration and disintegration of individual fibres before they showed any great degree of atrophy was the usual appearance in myopathy. There had evidently been a gross loss of fibres, and the presence of degenerating fibres indicated that the process of attrition was still going on in the muscle."

\section{Discussion}

The case here described is one of chronic progressive ophthalmoplegia occurring as an early sign of $\Omega$ muscular dystrophy. The condition is judged to be $N$ sufficiently rare to warrant description of a single $\mathrm{N}$ case, particularly as the histological findings in eye 0 muscle can be recorded in this instance. Unfor- 0 tunately the patient was unwilling to allow tissue to be removed from other muscles. It is suggested that, in obscure cases of chronic progressive ophthal- $T$ moplegia, muscle biopsy may sometimes prove useful $\bar{O}$ in making a diagnosis and in deciding if the paralysis is neurogenic or myogenic. The case examined by 
Gordon Holmes for McMullen and Hine (1921) is probably another example of myopathic external ophthalmoplegia, but confirmatory muscle biopsy is lacking. Nikitin (1929) has described a brother and sister with external ophthalmoplegia, together with weakness of limb and trunk muscles. This he believed was due to a muscular dystrophy. They were two of a sibship of four whose parents were first cousins. The diagnosis was unproven because muscle biopsy was not performed, and there were certain features present which were quite unlike those of a myopathy. For example the paresis was congenital and non-progressive, and in one of the patients there was actual improvement over a period of years. Elliott (1939) demonstrated a woman of 39 years who had chronic progressive ophthalmoplegia associated with weakness and wasting of other muscles. Ten years before, the eyelids had begun to droop, and six years later the eyeballs began to get fixed ; in another three years the muscles of the face, neck, shoulder girdle, and arms weakened and wasted. The wasting was thought to be myopathic. Martin (1939) described two children with external ophthalmoplegia who showed, in addition, myopathic features. One had weakness of nearly all the facial muscles, and the other showed generalized hypotonus of the somatic musculature. Prostigmin was without influence on the weakness. It seems probable that, in the group of cases of chronic progressive ophthalmoplegia which Wildbrand and Saenger separated and which has frequently been attributed to nuclear degeneration, the site of the lesion may often be not in the nervous system but in the muscles.

The cardiovascular findings in the present case suggest that the heart muscle may also be the site of myopathic degenerative changes. Marinesco (1910), in his monograph on the diseases of muscle, asserts that of the muscles which offer extraordinary resistance to invasion by muscular dystrophy, the heart is the most notable. Although rare, cardiac myopathy has sometimes been described, e.g. by Potter (1909), Globus (1923), and Berblinger and Duken (1929).

\section{Summary}

The pathology underlying the syndrome of chronic progressive ophthalmoplegia is discussed.

The value of biopsy material obtained from external ocular muscles is described as an aid to diagnosis.

A case is described in which histological examination of portions of external recti showed that chronic progressive ophthalmoplegia was of myopathic origin.

This subject also had bradycardia from branch bundle block, and the problem of whether there existed a cardiac myopathy is discussed.

\section{REFERENCES}

Altland, W. (1909). Arch. Ophthalmol., Chicago, 38, 296.

Ayres, S. C. (1896). Amer. J. Ophthalmol., 13, 65.

Beaumont, W. M. (1900). Trans. Ophthalmol. Soc. U.K., $20,258$.

Berblinger and Duken (1929). Z. Kinderheilk., 47, 1.

Bradburne, A. E. (1912). Trans. Ophthalmol. Soc., U.K., $32,142$.

Collins, E. T. (1909). Ibid., 29, 225.

Cooper, H. (1910). Brit. med. J., $1,917$.

Crigler, L. W. (1914). Arch. Ophthalmol., Chicago, 43, 268.

Crouzon, O. (1929). “Études sur les maladies familiales nerveuses et dystrophiques." Masson, Paris.

Elliott, F. A. (1939). Proc. roy. Soc. Med., 32, 876.

Füchs, E. (1890). Arch. Ophthalmologie, 36, 234.

Globus, J. H. (1923). Arch. Neurol. Psychiat., Chicago, $9,59$.

Marinesco, G. (1910). " Maladies des Muscles, Nouv. traite de méd et de thérapeutique," pp. 34 and 110. Fasc. 37. Paris.

Martin, J. P. (1939). Proc. roy. Soc. Med., 32, 876.

McMullen, W. H. (1912). Trans. Ophthalmol. Soc., U.K., 32,111 .

, and Hine, M. L. (1921). Brit. J. Ophthalmol., 5, 337.

Nikitin, M. P. (1929). Z. Ges. Neurol. Psychiat., 120, 575.

Potter, F. C. (1909). N.Y. med. J., 90, 398.

Wildbrand, H., and Saenger, A. (1921). Neurologie des Auges., 8, 121. Munich, Bergmann. 


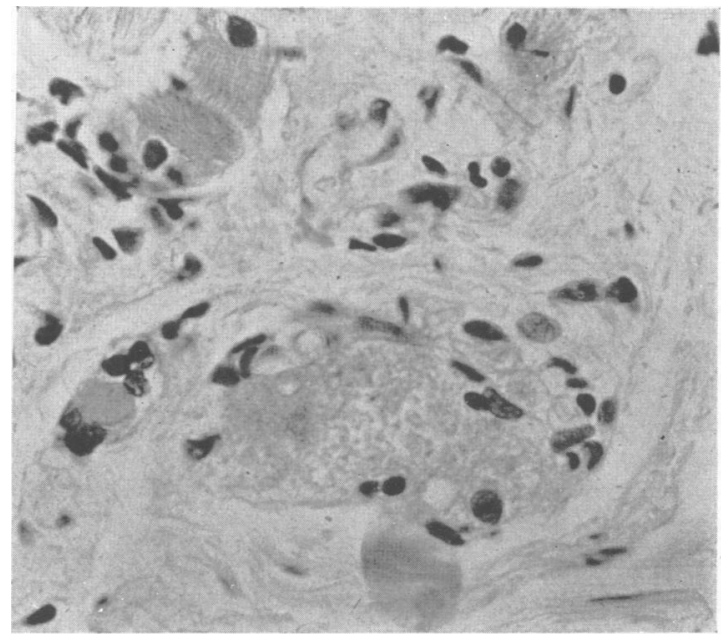

Fig. 1.-A swollen, granular muscle fibre with proliferated nuclei is seen cut in oblique section. Compare with two smaller normal fibres.

(Stained iron hæmatoxylin. van Giesen. $\times 500$.)

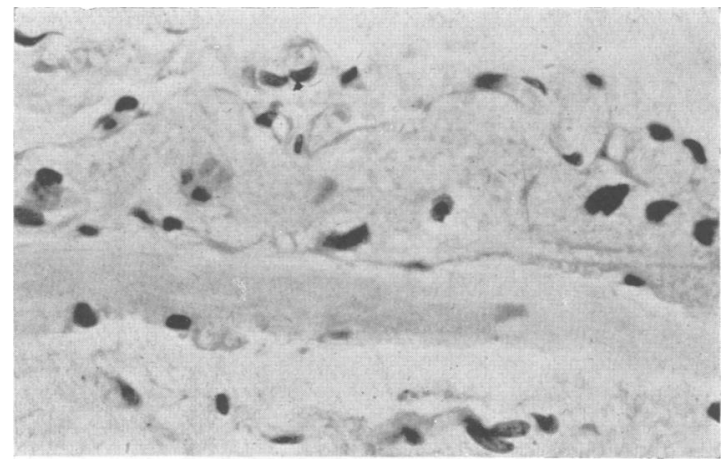

FIG. 2.-Irregularly swollen fibre showing granular disintegration and nuclear proliferation is seen alongside a normal fibre.

(Stained iron hæmatoxylin. van Giesen. $\quad \times 550$.)

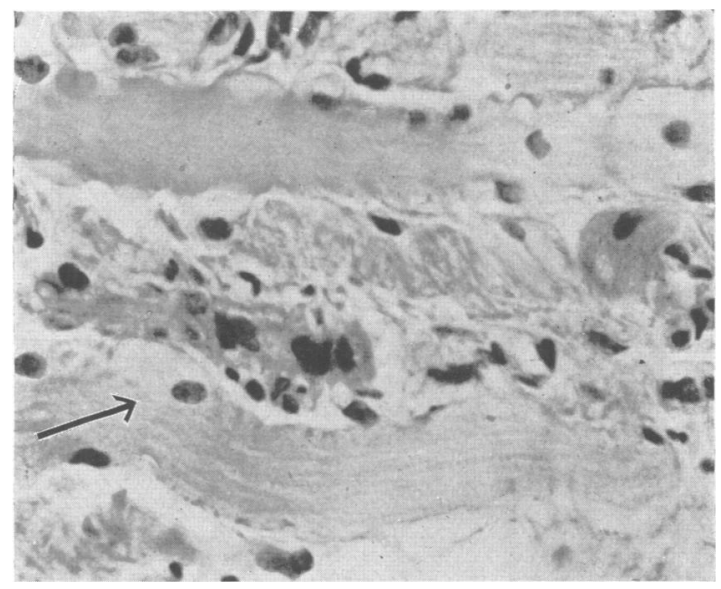

FIG. 3.-A shrunken fibre undergoing hyaline degeneration, with swollen, hyperchromatic internal nuclei, is seen between two normal fibres. One of these shows a pale granular oval area, indicated by arrow, under its sarcolemma.

(Stained iron hæmatoxylin. van Giesen. $\times 500$.) 